\title{
Contabilidad financiera y toma de decisiones empresariales
}

\author{
Financial accounting and business decision making
}

Contabilidade financeira e tomada de decisão empresarial

\section{ARTÍCULO GENERAL}

\begin{tabular}{|c|c|c|c|}
\hline $\begin{array}{c}\text { Domingo Hernandez-Celis } \\
\text { dhernández@unfv.edu.pe } \\
\text { https://orcid.org/0000-0002-9759-4436 } \\
\text { Universidad Nacional Federico Villarreal } \\
\text { Universidad Norbert Wiener } \\
\text { Lima - Perú }\end{array}$ & $\begin{array}{c}\text { Fernando Grimaldo Inocente Jacobe } \\
\text { https://orcid.org/0000-0001-6792-3131 } \\
\text { fernando.inocente@unmsm.edu.pe } \\
\text { Universidad Ricardo Palma } \\
\text { Universidad Nacional Mayor de San Marcos } \\
\text { Universidad Privada del Norte } \\
\text { Universidad Norbert Wiener } \\
\text { Lima - Perú }\end{array}$ & $\begin{array}{c}\text { Milagros Sandoval Ypanaqué } \\
\text { https://orcid.org/0000-0003-3631-029X } \\
\text { mili_1019@hotmail.com } \\
\text { Universidad Nacional Federico Villarreal } \\
\text { Lima - Perú }\end{array}$ & $\begin{array}{c}\text { Julia Paola Hernandez-Celis-Vallejos } \\
\text { 2020000068@unfv.edu.pe } \\
\text { https://orcid.org/0000-0001-6221-7422 } \\
\text { Universidad Nacional Federico Villarreal } \\
\text { Lima - Perú }\end{array}$ \\
\hline
\end{tabular}

Recibido 07 de Junio 2021 | Arbitrado y aceptado 25 de Agosto 2021 | Publicado en 04 Diciembre 2021

\section{RESUMEN}

El problema se ha identificado en la toma de decisiones de las grandes empresas comerciales de Lima Metropolitana. Esta problemática se expresa en la siguiente pregunta: $¿ D e$ qué forma la contabilidad financiera, podrá facilitar la toma de decisiones en las grandes empresas comerciales de Lima Metropolitana? Ante la problemática, se propone la solución a través de la formulación de la hipótesis: La contabilidad financiera facilita la toma de decisiones en las grandes empresas comerciales de Lima Metropolitana. Este trabajo se ha orientado al siguiente objetivo: Determinar la forma como la contabilidad financiera podrá facilitar la toma de decisiones en las grandes empresas comerciales de Lima Metropolitana. El resultado más importante es que el 80 por ciento de los encuestados acepta que la contabilidad financiera facilita la toma de decisiones en las grandes empresas comerciales de Lima Metropolitana.

Palabras clave: Contabilidad financiera; toma de decisiones $y$ grandes empresas comerciales de Lima Metropolitana.

\section{ABSTRACT}

The problem has been identified in the decision-making of large commercial companies in Metropolitan Lima. This problem is expressed in the following question: How can financial accounting facilitate decision-making in large commercial companies in Metropolitan Lima? Faced with the problem, the solution is proposed through the formulation of the hypothesis: Financial accounting facilitates decision-making in large commercial companies in Metropolitan Lima. This work has been oriented to the following objective: To determine the way in which financial accounting can facilitate decision-making in large commercial companies in Metropolitan Lima. The most important result is that 80 percent of those surveyed agree that financial accounting facilitates decision-making in large commercial companies in Metropolitan Lima.

Keywords: Financial accounting; decision-making and large commercial companies in Metropolitan Lima.

\section{RESUMO}

O problema foi identificado na tomada de decisões de grandes empresas comerciais na região metropolitana de Lima. Este problema é expresso na seguinte pergunta: Como a contabilidade financeira pode facilitar a tomada de decisões em grandes empresas comerciais na região metropolitana de Lima? Diante do problema, a solução é proposta através da formulação da hipótese: A contabilidade financeira facilita a tomada de decisões em grandes empresas comerciais na região metropolitana de Lima. Este trabalho foi orientado para o seguinte objetivo: Determinar a maneira pela qual a contabilidade financeira pode facilitar a tomada de decisões em grandes empresas comerciais na região metropolitana de Lima. O resultado mais importante é que $80 \%$ dos entrevistados concordam que a contabilidade financeira facilita a tomada de decisões em grandes empresas comerciais na região metropolitana de Lima.

Palavras-chave: Contabilidade financeira; tomada de decisão e grandes empresas comerciais na região metropolitana de Lima. 


\section{Introducción}

El problema se ha identificado en la toma de decisiones de las grandes empresas comerciales de Lima Metropolitana. Entre los síntomas o efectos se tiene que las grandes empresas comerciales de Lima Metropolitana, pese al esfuerzo que realizan sus directivos $\mathrm{y}$ funcionarios, no disponen de una adecuada estructura de financiamiento $\mathrm{o}$ endeudamiento. Según la Memoria Anual de la Cámara de Comercio de Lima y el Informe Anual de Macroconsult; se ha determinado que el 70\% de las empresas tienen deudas muy onerosas, el $67 \%$ de dichas empresas tiene mucha deuda corriente con trabajadores, SUNAT, proveedores, servicios públicos; el 62\% de las empresas disponen de un patrimonio mínimo lo que no les da la solvencia necesaria. Las empresas no disponen de un eficiente capital de trabajo, así como de una eficiente estructura de bienes de capital. El 58\% de las empresas afrontan problemas de capital de trabajo y el $70 \%$ ha incurrido en cuantiosas inversiones de bienes de capital que por el momento no representa problema, pero que a futuro por la movilidad de los negocios podría repercutir negativamente. Las empresas tienen ingresos que no son suficientes para los costos, gastos y tributos que genera su actividad. Los costos y gastos representan porcentajes sobre el $65 \%$ en el $70 \%$ de las empresas, lo que hace que disminuya la rentabilidad de dichas empresas. Las empresas no vienen siendo lo suficientemente eficientes, debido a una inadecuada racionalización de recursos. El 60\% de las empresas no aprovecha el talento humano; el 76\% de las empresas no racionaliza la electricidad, el agua y otros servicios. Las empresas no vienen logrando efectividad, debido a la falta de cumplimiento de metas y objetivos. Las empresas no tienen programas de mejoramiento de procesos y procedimientos empresariales. Las empresas no vienen logrando niveles adecuados de competitividad, por cuanto las empresas no sobresalen dentro de la economía regional ni nacional.

Las causas que han dado lugar a los síntomas o efectos son las siguientes: Las grandes empresas comerciales de Lima Metropolitana no disponen de departamentos, secciones o divisiones de contabilidad adecuadamente implementadas para que formulen periódicamente información financiera, económica y patrimonial para que los directivos puedan tomar decisiones informadas sobre endeudamiento, inversiones, ingresos, costos, gastos, tributos; y otros aspectos empresariales. No disponen de presupuestos financieros que les permitan medir a priori la liquidez que necesitan para entregar a los accionistas como dividendos o poder reinvertirla en las actividades de la empresa. En tanto cualquier 
resultado que obtengan no puede ser medido, porque no hay un instrumento de comparación; asimismo los resultados obtenidos tampoco se comparan con las empresas del mismo nivel y giro. Tampoco hacen comparaciones con la rentabilidad obtenida en ejercicios anteriores. Todo esto se configura como deficiencia en la decisión de dividendos o rentabilidad. Otra causa de la situación problemática es la deficiente identificación de riesgos en las actividades, procesos y procedimientos empresariales. No disponen de políticas tendentes a la eficiencia, efectividad, mejora continua y competitividad empresarial.

Pronostico: De continuar esta situación: Las grandes empresas comerciales de Lima Metropolitana continuarán teniendo una deficiente estructura de deudas lo que no les permitirá sacarle el máximo provecho a dicho endeudamiento. Continuarán teniendo una deficiente estructura de inversiones, lo que no les facilitará el máximo aprovechamiento de sus activos corrientes y no corrientes. Continuarán teniendo una deficiente estructura de ingresos, costos, gastos y tributos, con lo cual los niveles de rentabilidad serán mínimos e incluso obtendrían pérdida. No podrán sacarle el máximo provecho a los recursos que explotan. Continuarán no cumpliendo metas y objetivos. Continuarán sin procesos de mejoramiento continuo. Continuarán siendo incompetentes en el sector donde se desarrollan.

Control de pronóstico: Para solucionar la problemática presentada, debe tenerse en cuenta lo siguiente: Utilizar la contabilidad financiera, como herramienta de información para la planeación de actividades, procesos y procedimientos empresariales. Utilizar la contabilidad financiera, como herramienta para la toma de decisiones sobre endeudamiento e inversiones; ingresos, costos, gastos, tributos y resultados; actividades de operación, inversión y endeudamiento; cambios patrimoniales; etc. Utilizar la contabilidad financiera, junto a otras herramientas, para facilitar la eficiencia empresarial. Utilizar la contabilidad financiera, junto a otras herramientas, para facilitar la efectividad empresarial. Utilizar la contabilidad financiera, junto a otras herramientas, para facilitar la mejora continua empresarial. Utilizar la contabilidad financiera, junto a otras herramientas, para facilitar la competitividad empresarial.

Problema general: ¿De qué forma la contabilidad financiera podrá facilitar la toma de decisiones en las grandes empresas comerciales de Lima Metropolitana?

Problemas específicos: a) ¿De qué manera la información financiera 
podrá suministrar elementos para la toma de decisiones en las grandes empresas comerciales de Lima Metropolitana?; b) ¿De qué modo la información económica podrá proporcionar recursos para la toma de decisiones de las grandes empresas comerciales de Lima Metropolitana?; c) ¿De qué forma la información patrimonial podrá facilitar la toma de decisiones en las grandes empresas comerciales de Lima Metropolitana?

Objetivo general: Determinar la forma como la contabilidad financiera podrá facilitar la toma de decisiones en las grandes empresas comerciales de Lima Metropolitana.

Objetivos específicos: a) Establecer la manera como la información financiera podrá suministrar elementos para la toma de decisiones en las grandes empresas comerciales de Lima Metropolitana; b) Determinar el modo como la información económica podrá proporcionar recursos para la toma de decisiones de las grandes empresas comerciales de Lima Metropolitana; c) Establecer la forma como la información patrimonial podrá facilitar la toma de decisiones en las grandes empresas comerciales de Lima Metropolitana.

Justificación: En este trabajo en primer lugar se ha identificado la problemática en la toma de decisiones de las grandes empresas comerciales de Lima Metropolitana. Este trabajo permitirá que las grandes empresas comerciales de Lima Metropolitana utilicen los indicadores de la contabilidad financiera en la toma de decisiones sobre financiamiento, inversiones $\mathrm{y}$ variaciones patrimoniales.

Hipótesis principal: La contabilidad financiera facilita la toma de decisiones en las grandes empresas comerciales de Lima Metropolitana.

Hipótesis específicas: a) La información financiera suministra elementos para la toma de decisiones en las grandes empresas comerciales de Lima Metropolitana; b) La información económica proporciona recursos para la toma de decisiones de las grandes empresas comerciales de Lima Metropolitana; c) La información patrimonial facilita la toma de decisiones en las grandes empresas comerciales de Lima Metropolitana.

Investigaciones nacionales: Rondón (2013) aborda el estudio de la contabilidad financiera como ciencia que tiene un proceso que termina presentando los estados financieros y su utilidad como fuentes de información para la valoración de la empresa y 
la toma de decisiones. Álvarez (2015) resalta la relevancia de la contabilidad en la toma de decisiones sobre financiamiento, inversiones; ventas, costos, gastos y resultados. Fernández (2015), destaca que la contabilidad financiera facilita la efectividad de las empresas y que por tanto resulta adecuado obtener información contable razonable que permite obtener indicadores para las decisiones financieras y de otro tipo. Urbano (2015), destaca la relevancia de contar con una herramienta como la contabilidad en la generación de información financiera y económica para ser utilizada en la planeación, organización, dirección, coordinación y control empresarial.

Investigaciones internacionales: Lucano (2014) indica que la contabilidad se ve afectada por indicadores macroeconómicos como la inflación y que por tanto es necesario el planteo general del problema de la inflación en los informes financiero-contables en oposición al principio de costo histórico. León (2013) plantea un modelo contable externo para la administración local, la cual, al permitir el control y seguimiento por partida doble del global de acontecimiento de naturaleza presupuestaria generada en la Entidad a lo largo del ejercicio económico, adquiere la condición de integral. Fuentes (2014), aborda la información contable de las organizaciones sin fines de lucro, desde la perspectiva de su análisis y configuración en el marco conceptual. Martínez (2013), destaca la gran importancia de la contabilidad financiera en la gestión empresarial. Pereda (2013), presenta la contabilidad a través de los estados financieros y dice que permite obtener indicadores financieros que luego de ser analizados e interpretados facilitan la toma de decisiones sobre actividades de operación, inversiones y financiamiento. Rodas (2013), describe un conjunto de decisiones de financiamiento, que permiten realizar las inversiones que necesitan las empresas para desarrollarse en el marco de un mercado competitivo. Bossi (2015), señala que resulta relevante contar con la información de la contabilidad financiera para poder tomar decisiones tanto financieras como económicas.

\section{Bases teóricas:}

Contabilidad financiera empresarial: Según Anthony (2013), la historia no precisa una fecha exacta del surgimiento de la contabilidad, simplemente se da una aproximación y evidencia que ésta tiene sus orígenes en la aparición del comercio. Según Ferrer (2014); la contabilidad financiera empresarial es el proceso de reconocer, medir, registrar y presentar la información de las empresas en los estados financieros; los mismos que reflejan los efectos de las transacciones y otros sucesos de una empresa, agrupándolos por categorías, según sus características económicas, a los que se les llama elementos. 
Según Guajardo (2015) la contabilidad financiera empresarial comprende la valuación, registro y presentación de las transacciones; es una clase especial de contabilidad, cuyo objetivo es producir información dirigida a un grupo específico de interesados. Según Besteiro y Mazarracín (2015) la contabilidad financiera empresarial es el proceso constructivo de la información empresarial, que permite obtener los estados financieros. Según Horngren (2015) la contabilidad financiera, es la ciencia de la empresa, mediante la cual se recolectan, se clasifican, se registran, se sumarizan y se informa de las operaciones cuantificables en dinero, realizadas por una entidad económica. Según Diéguez (2015) la contabilidad financiera es una ciencia, una técnica y también un arte. Es ciencia porque tiene una metodología bien estructurada y ayuda a solucionar los problemas de las empresas. Anthony (2014) está de acuerdo con Meigs (2013), cuando indica que la contabilidad financiera es aquella actividad profesional que consiste en valuar, registrar y presentar las transacciones de compras, ventas, ingresos, egresos, provisiones, apertura y cierre y otras transacciones propias del giro de una empresa. La valuación consiste en determinar un valor para la transacción a nivel de su adquisición, venta o utilización y finalmente saldos según estados financieros. Según Montesinos (2015), la contabilidad financiera es el proceso que permite reconocer, medir, registrar y presentar la información de las empresas de acuerdo con normas especializadas. Según Bellido (2013), señala que los estados financieros incluyen un balance general, un estado de resultados o de ganancias y pérdidas, un estado de movimiento de las cuentas de patrimonio, un estado de flujo de efectivo y las notas a los estados financieros, así como otros estados y material explicativo que son parte integral de dichos estados. Según Bellido (2013), los ratios de liquidez mide la capacidad de pago que tiene la empresa para hacer frente a sus deudas de corto plazo. Los ratios de gestión o actividad miden la efectividad y eficiencia de la gestión, en la administración del capital de trabajo, expresan los efectos de decisiones y políticas seguidas por la empresa, con respecto a la utilización de sus fondos. Los ratios de solvencia, endeudamiento o apalancamiento, muestran la cantidad de recursos que son obtenidos de terceros para el negocio. Los ratios de rentabilidad miden la capacidad de generación de utilidad por parte de la empresa. Para Flores (2016), la contabilidad financiera culmina con la formulación de los estados financieros de las empresas; para lo cual se deben tener en cuenta las Normas Internacionales de Contabilidad.

Toma de decisiones empresariales: Según Robbins y Coulter (2013), la toma de 
decisiones empresariales es la llave final de todo el proceso administrativo: ningún plan, ningún control, ningún sistema de organización tiene efecto, mientras no sé de una decisión. Para Stoner, Freeman y Gilbert (2013), la toma de decisiones en una organización invade cuatro funciones administrativas que son: planeación, organización, dirección y control. De los procesos existentes para la toma de decisiones, el proceso racional de toma de decisiones es catalogado como "el proceso ideal". Según Terry (2013), en la toma de decisiones la experiencia es un elemento clave puesto que las decisiones deben tomarse sobre una realidad altamente compleja debido al enorme número de variables que entran en juego. Según Koontz \& O`Donnell (2013), la toma de decisiones es importante porque mediante el empleo de un buen juicio, la toma de decisiones nos indica que un problema o situación es valorado y considerado profundamente para elegir el mejor camino a seguir según las diferentes alternativas y operaciones. Según Chiavenato (2013), el enfoque clásico de la administración trata del proceso administrativo y la organización racional del trabajo. La escuela de comportamiento humano, conocida también como la escuela de las elaciones humanas misma otorgaron mayor importancia a la persona. La teoría matemática aplicada a la solución de los problemas administrativos se conoce como Investigación de operaciones (IO). Según Flores (2016) las finanzas empresariales se refieren a las inversiones, deudas, ingresos, costos, gastos y resultados correspondientes. Según el IAI-España (2015), el control en el marco del Informe COSO es un instrumento de gestión que se utiliza para proporcionar una garantía razonable del cumplimiento de los objetivos.

Grandes Empresas Comerciales: Según Rodríguez (2013), las grandes empresas comerciales son unidades económicas constituidas por una persona jurídica, bajo cualquier forma de organización o gestión empresarial contemplada en la legislación vigente, que tiene como objeto la comercialización de bienes. Según Argibay (2013), las empresas desarrollan sus actividades y llevan a cabo una serie de transacciones como compras, ventas, cobros, pagos por mencionar lo elemental; todas esas transacciones con consideradas por la contabilidad financiera y de cuyo tratamiento se obtienen los estados financieros que sirven de base para la toma de decisiones. Analizando a Pérez (2014), las empresas deben crear y luego dirigir, toda una serie de relaciones entre su empresa y sus trabajadores, proveedores, bancos y clientes. Analizando a Ortega y Pacherres (2013); según la Ley 26887, Ley General de Sociedades, quienes constituyen la Sociedad convienen en aportar bienes o servicios para el ejercicio en común de actividades 
económicas, asimismo toda sociedad debe adoptar alguna de las formas previstas en esta ley. Según Flores (2016) las mercaderías de las empresas comerciales son bienes muebles que están gravados con tributos. Según Bahamonde (2016); el Impuesto General a las Ventas grava las venta en el país de bienes muebles (mercaderías). Analizando a Bernal y Espinoza (2014); el Impuesto a la Renta grava las rentas que provengan del capital, del trabajo y de la aplicación conjunta de ambos factores. Analizando a Buendía (2016); el Impuesto Temporal a los Activos Netos es un impuesto al patrimonio, que grava los activos netos como manifestación de capacidad contributiva, dicho impuesto utilizarse como crédito contra los pagos a cuenta y de regularización del Impuesto a la Renta. Según Flores (2016); la contabilidad se registra utilizando una herramienta especial denominada Plan Contable General Empresarial el mismo que está armonizado con las Normas internacionales de Información financiera. Para Chapi (2016) la contabilidad registra todas las transacciones de las empresas, tales como operaciones de activos, pasivos, ingresos y gastos; dichas transacciones son reconocidas, medidas, anotadas y presentadas y reveladas en los reportes especiales. Para Sáenz (2016) las cuentas contables se encuentran afectadas por el intensivo proceso de revisión de las normas contables vigentes, y por el desarrollo de nuevas formas y tipos de negocios. Según Herrera (2014) un plan o catálogo de cuentas es una herramienta necesaria para procesar información contable. Según Omeñáca (2015) las Normas internacionales de información financiera establecen los requisitos de reconocimiento, medición, presentación e información a revelar, respecto de hechos y estimaciones de carácter económico. Según Ferrer (2014) los estados financieros de las empresas son el producto final de la contabilidad financiera. De otro lado, según Apaza (2015) los estados financieros reflejan los efectos de las transacciones y otros sucesos de una empresa, agrupándolos por categorías, según sus características económicas, a los que se les llama elementos. Según Aguilar (2015); debe reconocerse toda partida que cumpla la definición de elemento siempre que: sea probable que cualquier beneficio económico asociado con la partida llegue a, o salga de la empresa; $\mathrm{y}$, la partida tenga un costo o valor que pueda ser medido con fiabilidad.

\section{Método}

Tipo de la investigación: Esta investigación es de tipo aplicada. Por cuanto se propone a las grandes empresas comerciales de Lima Metropolitana que apliquen la contabilidad financiera, para disponer de información que les permita tomar decisiones bien informadas sobre los activos, pasivos, 
patrimonio, ingresos y gastos: así como la liquidez, gestión, solvencia y rentabilidad de dichas empresas.

Diseño utilizado en el estudio: El diseño que se aplicó fue el no experimental. El diseño no experimental se define como la investigación que se realizó sin manipular deliberadamente la contabilidad financiera y la toma de decisiones en las grandes empresas comerciales de Lima Metropolitana.

Población: Al respecto se ha tomado como fuente a la base de Principales Contribuyentes (PRICOS) de la Intendencia Lima (IL) de la Superintendencia Nacional de Aduanas y Administración tributaria (SUNAT). Para la SUNAT, un PRICO es una empresa grande cuyos ingresos brutos anuales son iguales o mayores a 3,000 Unidades Impositivas Tributarias (UIT). Para el 2016, la UIT fue igual a 3,950 Soles. De este modo se considera un PRICO a la gran empresa que tenga ingresos iguales o superiores a 11'850,000.00 Soles (esto en promedio es casi un millón de soles mensuales). A Febrero del 2016 se tuvo el siguiente universo de PRICOS o grandes empresas: a) 3,643 PRICOS a nivel nacional: $100 \%$; b) 1,937 PRICOS de la Intendencia Lima de la SUNAT: 53\% y c) 106 PRICOS comerciales de la Intendencia Lima de la SUNAT: 5\%. De este modo la población de investigación estuvo conformada por 106 PRICOS o grandes empresas comerciales y de las mismas 140 personas que fueron trabajadores.

Muestra: La muestra estuvo conformada por 10 grandes empresas y como representantes de las mismas 100 personas relacionadas con la contabilidad financiera empresarial y la toma de decisiones.

\section{Resultados}

Resultado 1: El 80\% de los encuestados acepta que la contabilidad financiera proporciona información razonable de las empresas. Contabilidad financiera es la rama de la contabilidad que se encarga de recolectar, clasificar, registrar, resumir e informar sobre las operaciones valorables en dinero realizadas por un ente económico. Su función principal es llevar en forma histórica la vida económica de una empresa. También se conoce como contabilidad externa o general y, en conjunto con la contabilidad administrativa y la contabilidad de costos, compone la estructura contable típica de toda organización. Es un área de la contabilidad que tiene como objetivo preparar y elaborar información contable destinada a los usuarios externos. Dicha información está sujeta a 
una regulación externa a la empresa (del estado, de organizaciones profesionales, etc.).

Resultado 2: E1 82\% de los encuestados acepta que la liquidez es la disponibilidad de recursos financieros en el corto plazo. La liquidez es la capacidad de la empresa de hacer frente a sus obligaciones de corto plazo. La liquidez se define como la capacidad que tiene una empresa para obtener dinero en efectivo. Es la proximidad de un activo a su conversión en dinero. Para medir la liquidez de una empresa se utiliza el ratio o razón de liquidez. La cual mide la capacidad de la empresa para hacer frente a sus obligaciones de corto plazo. Del análisis de estas razones se puede conocer la solvencia de efectivo de la empresa y su capacidad de permanecer solvente en caso de acontecimientos adversos.

Resultado 3: El 84\% de los encuestados acepta que la liquidez general se obtiene relacionando el activo corriente con el pasivo corriente.

Resultado 4: El 65\% de los encuestados acepta que la rentabilidad sobre ventas es la relación de la utilidad neta sobre las ventas.

Resultado 5: E1 76\% de los encuestados acepta que la rentabilidad sobre activos es la relación entre la utilidad neta y el activo total.

Resultado 6: E1 77\% de los encuestados acepta que la rentabilidad sobre el patrimonio es la relación entre la utilidad y el patrimonio neto.

Resultado 7: E1 78\% de los encuestados acepta que los indicadores de gestión comprenden la rotación de inventarios, cuentas por cobrar, cuentas por pagar y otros ratios de la operatividad empresarial.

Resultado 8: El 67\% de los encuestados acepta que la rotación de inventarios mide el número de veces que se cambia el inventario en un determinado período.

Resultado 9: El 86\% de los encuestados acepta que el mayor valor de la rotación de inventarios indica eficiencia y efectividad en la administración de dicho rubro.

Resultado 10: Este resultado favorece el modelo de investigación desarrollado; por cuanto el $87 \%$ de los encuestados acepta que la rotación de cuentas por cobrar mide el número de veces que cobran las empresas. 
Resultado 11: El 90\% de los encuestados acepta que el mayor valor de la rotación de cuentas por cobrar indica efectividad de las políticas de cobranza en un determinado periodo.

Resultado 12: Este resultado favorece el modelo de investigación desarrollado; por cuanto el $81 \%$ de los encuestados acepta que la solvencia se mide por la relación del patrimonio neto con el pasivo.

Resultado 13: El 80\% de los encuestados acepta que la toma de decisiones es el proceso que permite elegir entre varias alternativas, la mejor para las grandes empresas.

Resultado 14: El 78\% de los encuestados acepta que las decisiones financieras pueden ser de inversión, financiamiento, rentabilidad y riesgos.

Resultado 15: El 92\% de los encuestados acepta que las decisiones de inversión están relacionadas con el capital de trabajo y los bienes de capital.

Resultado 16: El 76\% de los encuestados acepta que las decisiones de financiamiento están relacionadas con el endeudamiento propio y de terceros.

Resultado 17: El 84\% de los encuestados acepta que las decisiones sobre rentabilidad tienen que ver con la propensión o aversión al riesgo.

Resultado 18: El 85\% de los encuestados acepta que las decisiones operativas están relacionadas con las actividades y procesos.

El Resultado 19: $86 \%$ de los encuestados acepta que las decisiones operativas se relacionan con el financiamiento e inversiones que realizan.

Resultado 20: El 74\% de los encuestados acepta que las decisiones patrimoniales se relacionan con el incremento y reducción del capital social, las reservas y utilidades.

Contrastación de hipótesis: La hipótesis estadística es una afirmación respecto a las características de la población. Contrastar una hipótesis es comparar las predicciones realizadas por el investigador con la realidad observada. Si dentro del margen de error que se ha admitido 5.30\%, hay coincidencia, se acepta la hipótesis y en caso contrario se rechaza. Este es el criterio fundamental para la contrastación.

La media o valor promedio de la variable independiente contabilidad financiera empresarial es $80.12 \%$ en cambio la media o promedio de la 
variable dependiente toma de decisiones empresariales es $80.46 \%$. Lo que indica un buen promedio para ambas variables, siendo mejor para la variable dependiente, que es la que se busca solucionar, lo cual apoya el modelo de investigación llevado a cabo. De otro lado, la desviación típica mide el grado de desviación de los valores en relación con el valor promedio, en este caso es $4.39 \%$ para la variable independiente contabilidad financiera empresarial y $4.12 \%$ para la variable dependiente toma de decisiones empresariales, lo que quiere decir que hay alta concentración en los resultados obtenidos; siendo mejor dicha concentración en la variable dependiente, lo que favorece al modelo de investigación propuesto. La correlación es igual a $80.90 \%$, lo cual indica correlación directa (positiva), regular, por tanto, aceptable. La prueba de significancia estadística busca probar que existe una diferencia real, entre dos variables estudiadas, y además que esta diferencia no es al azar. El valor de significancia, igual a $4.30 \%$, el mismo que es menor al margen de error propuesto del $5.30 \%$, lo que, de acuerdo con la teoría estadística generalmente aceptada, permite rechazar la hipótesis nula y aceptar la hipótesis alternativa. La regresión es una técnica estadística generalmente aceptada que relaciona la variable dependiente toma de decisiones empresariales con la información suministrada por otra variable independiente contabilidad financiera empresarial. El Coeficiente de Determinación Lineal $(\mathrm{R}$ cuadrado $=80.10 \%$ explica que el $80.10 \%$ de la variación total se debe a la variable independiente: contabilidad financiera empresarial.

\section{Discusión de resultados}

La discusión de los principales resultados es la siguiente:

1) El $85 \%$ de los encuestados acepta que la contabilidad financiera proporciona información razonable de las empresas. Este resultado es similar al $84 \%$ presentado, aunque en otra dimensión espacial y temporal, por Fernández (2015). La contabilidad financiera como instrumento para la efectividad empresarial. Ambos resultados son razonables y favorecen la investigación desarrollada.

2) El $75 \%$ de los encuestados acepta que la toma de decisiones es el proceso que permite elegir entre varias alternativas, la mejor para las grandes empresas. Este resultado es similar al 73\% presentado, aunque en otra dimensión espacial y temporal, por Fernández (2015). La contabilidad 
financiera como instrumento para la efectividad empresarial. Ambos resultados son razonables y favorecen la investigación desarrollada.

\section{Conclusiones}

Las conclusiones son las siguientes:

a) Se ha determinado que la contabilidad financiera facilita la toma de decisiones; mediante la información financiera, información económica e información patrimonial de dichas organizaciones.

b) Se ha establecido que la información financiera facilita la toma de decisiones; mediante la información sobre inversiones, deudas, rentabilidad y riesgos de dichas entidades.

c) Se ha determinado que la información económica facilita la toma de decisiones; mediante la información sobre ventas, costos, gastos y resultados de dichas entidades.

d) Se ha establecido que la información patrimonial facilita la toma de decisiones; mediante la información de las variaciones del capital social, excedente de revaluación, reservas y resultados acumulados de dichas empresas.

e) Se ha determinado que la contabilidad financiera es relevante; sin embargo, para efectos de toma de decisiones, los directivos deberían complementarse con información económica, administrativa, financiera, estadística, legal, laboral, tributaria, etc., de tal modo que se tomen decisiones bien informadas.

\section{Recomendaciones}

Las recomendaciones son las siguientes:

1) Se recomienda tener en cuenta que la contabilidad financiera facilita la toma de decisiones. Por tanto, se debería capacitar, entrenar y perfeccionar al personal contable para que formule la contabilidad financiera razonable de dichas entidades.

2) Se recomienda tener en cuenta que la información financiera facilita la toma de decisiones. Por tanto, se debería analizar estructuralmente, horizontalmente y aplicar los ratios financieros para comprender la situación y tomar decisiones bien informadas. 
3) Se recomienda tener en cuenta que la información económica facilita la toma de decisiones. Por tanto, se debería hacer comparaciones internas como externas para obtener información de calidad para la toma de decisiones.

4) Se recomienda tener en cuenta que la información patrimonial facilita la toma de decisiones, Por tanto, se debería ponderar la información patrimonial para retribuir adecuadamente a los accionistas por los riesgos que corren en este tipo de empresas.

5) Se recomienda tener en cuenta que la contabilidad financiera solo es una parte de la información empresarial. Por tanto, se debería disponer de otros tipos de información para poder complementar, tales como información económica, financiera, administrativa, estadística, legal, laboral, tributaria, etc, de tal modo que se tomen decisiones bien informadas.

\section{Referencias}

Anthony, R. (2013). Accounting and Business Administration. Mc Graw Hill.

Apaza, M. (2015). Estados financieros. Instituto Pacífico.

Argibay, M. (2013). Contabilidad financiera y administración. Ideas propias Editorial

Bahamonde, H. (2016). Tributación y administración empresarial. Editorial San Marcos.

Bellido, P. (2013). Administración Financiera. Editorial Técnico-Científica SA.

Bernal, J. y Espinoza, C. (2014). Impuesto a la Renta. Actualidad empresarial.

Besteiro, M. y Mazarracín, M. (2015). Contabilidad Financiera Superior. Editorial Pirámide.

Bossi, A. (2015). Medición del capital intelectual en el sector público para efectos de tomar decisiones. [Tesis doctoral, Universidad Autónoma de México]. Repositorio institucional.

Buendía, A. (2016). Tributos empresariales. Editorial Santo Domingo.

Chapi, A. (2016). Contabilidad y estados financieros. Actualidad empresarial Chiavenato, I. (2013). Teoría General de la Administración.: Mc Graw Hill Interamericana Editores, SA de CV. 
Diéguez, J. (2015). Contabilidad Financiera. Pirámide.

Fernández, H. (2015). La contabilidad financiera como instrumento para la efectividad empresarial. [Tesis doctoral, Universidad Nacional Federico Villarreal]. Repositorio institucional

Ferrer, A. (2014). Formulación, análisis e interpretación de los estados financieros. Editorial Ferrer EIRL.

Flores, J. (2016). Contabilidad financiera. Centro de Especialización en Contabilidad y Finanzas-CECOF Asesores.

Flores, J. (2016). Gestión Financiera, tributación y administración de empresas. CECOF Asesores.

Flores, J. (2016). Plan contable General para empresas. Editado por Actualidad empresarial

Fuentes, M. (2014). Las entidades no lucrativas: su información contable desde el enfoque del marco conceptual. [Tesis doctoral, Universidad de Barcelona]. Repositorio institucional.

Gómez, L. (2014). Contabilidad. Editorial Ramos.

Guajardo, G. (2014). Contabilidad Financiera. Mc Graw Hill

Horngren, C. (2015). Contabilidad financiera: Editorial: Pearson

Instituto de Auditores Internos de España (2015). Control interno en el marco del Informe Coso. Editorial Días de Santos.

Koontz, H. \& O’Donnell, C. (2013). Administración Moderna. Litográfica Ingramex S.A.

León, J. (2013). Un modelo contable externo integral para las entidades locales. [Tesis doctoral, Universidad de Salamanca]. Repositorio institucional.

Lucano, P. (2014). El proceso inflacionario en la información financierocontable. [Tesis doctoral, Universidad de Barcelona]. Repositorio institucional.

Martínez, F. (2013). La revelación contable en la salamanca histórica. [Tesis doctoral, Universidad de Salamanca]. Repositorio institucional. 
Meigs, C. (2013). Contabilidad la base para decisiones gerenciales. Mcgraw-Hill

Montesinos, V. (2015). Contabilidad Financiera. Pirámide.

Omeñáca, J. (2015) Contabilidad General. Editorial Deusto.

Ortega, R. y Pacherres, A. (2013). Ley General de Sociedades. Editorial Tinco SAC.

Pereda, J. (2013). Teoría unitaria para el tratamiento de la información económica-financiera del grupo de empresas públicas. [Tesis doctoral, Universidad Complutense de Madrid]. Repositorio institucional.

Pérez, E. (2014). Organización y Administración de empresas comerciales en el Perú. Editorial San Marcos

Robbins, S., \& Coulter, M. (2013). Administración. Pearson Educación.

Rodas, M. (2013). Decisiones financieras para el desarrollo de las empresas industriales mexicanas. [Tesis doctoral, Universidad Autónoma de México]. Repositorio institucional

Rodríguez, L. (2013). Planificación y dirección de las empresas comerciales. Editorial Continental SA.

Rondón, J. (2013). Utilidad de los estadosfinancieros para la valoración de empresas. [Tesis doctoral, Universidad Nacional Federico Villarreal]. Repositorio institucional

Sáenz, A. (2016). Contabilidad. Editado Entrelíneas.

Stoner, J; Freeman, R., \& Gilbert, D. (2013). Administración. Prentice-Hall Hispanoamericana SA.

Terry, G. (2013). Administración. Prentice-Hall Hispanoamericana SA.

Urbano, R. (2015). Optimización presupuestaria a través del balance y la contabilidad matricial. [Tesis doctoral, Universidad Nacional Federico Villarreal]. Repositorio institucional. 